\title{
Control de indicadores para mejorar la gestión en redes de salud
}

\author{
Jin Morgan Angulo Infante \\ jinangulo@hotmail.com \\ Escuela de posgrado \\ Universidad Cesar Vallejo \\ ORCID: 0000-0001-7085-7454 \\ José Manuel Delgado Bardales \\ jmdelgadob@ucvvirtual.edu.pe \\ Escuela de posgrado \\ Universidad César Vallejo \\ Tarapoto - Perú \\ ORCID: 0000-0001-6574-2759 \\ Scopus autor ID: 24070333700 \\ Código Renacyt: P0050554
}

\section{RESUMEN}

El presente estudio de investigación determinó el control de indicadores de mejora de la gestión en las redes de salud. La investigación es de tipo descriptiva propositiva, y el estudio es tiene como diseño de investigación de tipo transversal no experimental, porque parte de la descripción del problema para dar una solución, sin hacer ninguna prueba de experimentación a dicha solución propuesta. En relación al control de indicadores; En estos tiempos se viene notando que los gobiernos regionales, están poniendo interés en el sector salud y se vienen innovando sus herramientas de gestión, como son el uso de indicadores, que cuyo control ayudará a tener una mejor administración en la Redes de Salud. Pero penosamente, hay, contextos que imposibilitan que la gestión de los directores de Red sea eficiente, debido a que se identificó nudos críticos a nivel de cada uno de los seis componentes que se trabajan en salud: gobernanza, financiamiento, provisión de servicios, recursos humanos, medicamentos, y sistema de información. Por eso es fundamental el análisis de la Salud de nuestra población porque constituye un elemento fundamental en el proceso de decidir la manera más eficiente de utilizar los recursos tanto humanos como financieros para mejorar la salud de las mismas, el monitoreo de los indicadores sanitarios, tienen como objetivo presentar un conjunto mínimo estandarizados de datos e información necesaria para caracterizar la situación de salud en nuestra región.

Palabras clave: indicador, gestión, herramientas de gestión, monitoreo. 


\title{
Control of indicators to improve management in health networks
}

\begin{abstract}
The present research study determined the control of management improvement indicators in health networks. The research is of a descriptive propositional type, and the study has a nonexperimental cross-sectional research design, because it starts from the description of the problem to give a solution, without doing any experimental test to said proposed solution. In relation to the control of indicators; In these times it has been noted that regional governments are putting interest in the health sector and their management tools are being innovated, such as the use of indicators, whose control will help to have a better administration in the Health Networks. But sadly, there are contexts that make it impossible for the Network directors' management to be efficient, due to the fact that critical nodes were identified at the level of each of the six components that work in health: governance, financing, provision of services, resources humans, drugs, and information system. That is why the analysis of the Health of our population is essential because it constitutes a fundamental element in the process of deciding the most efficient way to use both human and financial resources to improve their health, the monitoring of health indicators, have The objective is to present a minimum standardized set of data and information necessary to characterize the health situation in our region.
\end{abstract}

Keywords: indicator, management, management tools, monitoring.

Artículo recibido: 03 nov. 2020

Aceptado para publicación: 07 dic. 2020

Correspondencia jinangulo@ hotmail.com

Conflictos de Interés: Ninguna que declarar 


\section{INTRODUCCIÓN}

El control de indicadores es una herramienta del monitoreo y evaluación que nos permite establecer en qué punto nos encontramos frente al cumplimiento de las metas, es decir del valor final del indicador determinando en qué momento se cumplió con el objetivo tanto para instituciones públicas como privadas a nivel regional, nacional e internacional. Sin embargo, ello no se viene realizando por lo cual no se cuenta con información relevante para tomar decisiones correctivas o preventivas, de ser necesario. Si no se mide lo que se hace, no se puede controlar y si no se puede controlar, no se puede dirigir y si no se puede dirigir no se puede mejorar. Por lo tanto, si no llevamos un control de los indicadores, puede contribuir a que la institución tenga una deficiente gestión, ya que es en base a la evaluación de estos indicadores que se determina como resultado la eficiente o deficiente gestión administrativa de una organización.

Sin embargo, en los países europeos, la realidad es distinta, porque ellos trabajan sobre los valores y principios comunes de los sistemas sanitarios de la Unión Europea (2006/C 146/01) destacando, como valores esenciales, la universalidad, el acceso a una atención sanitaria de calidad, la equidad y la solidaridad. Los principios comunes contemplan la calidad, la seguridad, la atención basada en las pruebas y en la ética, la participación del paciente, el derecho a reparación, a la intimidad y a la confidencialidad. De hecho, el texto de la Directiva de Servicios Sanitarios Transfronterizos de la UE tiene como objeto establecer un marco general para la provisión de una atención sanitaria segura, de alta calidad y eficiente que se aplicará a la prestación de la atención sanitaria sin importar el modo en que se organiza, se provee y se financia o si es pública o privada.

En el Perú, el ministerio de salud organizado de acuerdo a su estructura, tienen un conjunto de problemas en cuanto a la gestión administrativa en la Redes de Salud a nivel nacional, esto por el motivo que todavía se sigue eligiendo a los directores de las Redes de Salud, por invitación política ya que es un cargo de confianza de los gobiernos regionales, y no por un concurso de méritos. Según Aliaga, R. (2016), no basta, por lo tanto, con definir buenos indicadores y metas realizables, debe existir un proceso de seguimiento que permita a los interesados saber si esas metas se van a alcanzar.

La Red de Salud Picota, que se encuentra en la Provincia de Picota, región San Martín, es la institución encargada de administrar los servicios de salud de la provincia mencionada, 
lastimosamente es una de las 10 Redes de Salud en la región San Martin, que está mal administrada, ya que no cuenta con personal idóneo que cumplan de manera óptima las funciones de gestión administrativa, conduciendo así a cometer actos irregulares dentro de la institución. Todo esto conlleva a una mala atención a los pacientes dentro de los establecimientos de salud. Aliaga, R. (2016); además el control de indicadores plantea la necesidad de establecer una metodología de reporte y seguimiento, para mejorar la gestión administrativa.

\section{ESTRATEGIAS METODOLÓGICAS o MATERIALES Y MÉTODOS}

El tipo de investigación es básica de diseño no experimental descriptivo con revisión sistemática, las revisiones sistemáticas son resúmenes de investigaciones científicas disponibles en las que las unidades de análisis son los estudios originales primarios, se constituyen en una herramienta esencial para sintetizar la información científica disponible, incrementar la validez de las conclusiones de estudios individuales e identificar áreas de incertidumbre donde sea necesario realizar investigación (Hernández, 2016, p.217).

Es básico no experimental porque se recogió información tal y como se la encontró sin aplicar ningún programa durante el proceso, no se manipularon las variables y es descriptivo porque da a conocer el control de indicadores para mejorar la gestión en redes de salud (Tamayo y Tamayo, 2003. p.46). Por lo mencionado, se revisó información científica que mostró como la ejecución del control de indicadores para mejorar la gestión redes de salud.

La recolección de datos se realizó a través de una tabla de registro de datos y la fuente fue la revisión bibliográfica de artículos científicos nacionales como internacionales que tuvieron como tema el control de indicadores para mejorar la gestión en redes de salud, se seleccionaron los 10 artículos más importantes según el nivel de evidencia de los que se tuvo acceso al artículo completo de donde se recolectaron la información necesaria que permitieron el análisis.

La técnica de análisis se desarrolló a través de la revisión sistemática, analizando cada uno de los artículos seleccionados para establecer una comparación de los resultados y conclusiones que permita conocer cómo está el nivel de implementación y ejecución del control de indicadores para mejorar la gestión en redes de salud, los avances de los mismos en el tiempo como las similitudes entre ellas. 


\section{RESULTADOS Y DISCUCIONES: RESULTADOS}

\begin{tabular}{|c|c|c|c|c|}
\hline \multicolumn{5}{|c|}{ DATOS DE LA PUBLICACION } \\
\hline 1. Autor & Año & $\begin{array}{l}\text { Nombre de la } \\
\text { investigación }\end{array}$ & Revista donde se ubica la publicación & onde se ubica la publicación \\
\hline Huerta, Patricia & $\begin{array}{r}\text { Anális } \\
\text { sa }\end{array}$ & $\begin{array}{l}\text { sis de los indicadores } \\
\text { anitarios chilenos }\end{array}$ & \multicolumn{2}{|c|}{$\begin{array}{cc}\text { http://revistas.ujat.mx/index.php/ } & \text { HORIZONTE SANITARIO; Vol. 18, } \\
\text { horizonte/article/view/2751 } & \text { Núm. } 2(2019) ; 2007-7459 ; 1665- \\
& 3262 ; 10.19136 / \mathrm{hs} . \mathrm{a} 18 \mathrm{n} 2\end{array}$} \\
\hline \multicolumn{5}{|c|}{ CONTENIDO DE LA PUBLICACIÓN } \\
\hline $\begin{array}{c}\text { Tipo de } \\
\text { Investigación }\end{array}$ & $\begin{array}{l}\text { Población y } \\
\text { Muestra }\end{array}$ & Instrumentos & $\begin{array}{c}\text { Aspectos } \\
\text { ético }\end{array}$ & Resultados \\
\hline Descriptivo & $\begin{array}{l}75 \text { Indicadores } \\
\text { Básicos de Salud } \\
\text { (IBS) utilizados } \\
\text { por los } \\
\text { establecimientos } \\
\text { de salud, } \\
\text { pertenecientes a } \\
\text { las redes } \\
\text { asistenciales de } \\
\text { los Servicios de } \\
\text { Salud en Chile. }\end{array}$ & $\begin{array}{l}\text { Estudio de } \\
\text { correspondencias } \\
\text { múltiples }\end{array}$ & $\begin{array}{l}\text { Sin } \\
\text { consentimie } \\
\text { nto porque es } \\
\text { de acceso } \\
\text { público }\end{array}$ & \begin{tabular}{lllr} 
Los indicadores de riesgos para la salud se & \multicolumn{2}{l}{ Debido al impacto en la } \\
caracterizaron principalmente porque en un & población, & todo \\
$50 \%$ y más de las regiones analizadas & indicador de gestión en \\
obtuvieron un resultado similar al resultado & salud debería & ser \\
esperado. A diferencia de los indicadores de & considerado por los & los \\
estadísticas vitales, en donde un $49 \%$ menos & directivos de las redes \\
de las regiones tuvieron un resultado similar al & sanitarias para guiar las \\
esperado en el mismo periodo. A la vez, los & estrategias & a \\
indicadores demográficos se asociaron a un & implementar en los \\
resultado esperado positivo y al contrario los & establecimientos & que \\
indicadores de acceso a la atención se & conforman las redes \\
asociaron a un resultado esperado negativo, en & asistenciales de & salud \\
el periodo analizado. & pública. &
\end{tabular} \\
\hline \multicolumn{5}{|c|}{ DATOS DE LA PUBLICACIÓN } \\
\hline 2. Autor & Año & bre de la investigac & ón Revista c & donde se ubica la publicación $\quad$ Volumen y número \\
\hline \multicolumn{2}{|c|}{$\begin{array}{l}\text { Cerdá Suárez, L.; } \\
\text { Santibáñez Vivanco, } \\
\text { A; Asensio del Arco, } \\
\text { E; Martínez A. }\end{array}$} & \multicolumn{3}{|c|}{$\begin{array}{lll}\text { Indicadores para mejorar la atención } & \text { https://doi.org/10.11144/ } & \text { Rev. Gerenc. Polit. Salud. 2018;17(35). } \\
\text { a pacientes según lean-seis-sigma: el } & \text { Javeriana.rgps17-35.abcd } & \\
\text { caso del } & & \\
\text { hospital Gustavo Fricke (Chile) } & \end{array}$} \\
\hline
\end{tabular}

\section{CONTENIDO DE LA PUBLICACIÓN}




\begin{tabular}{cccccc}
\hline $\begin{array}{c}\text { Tipo de } \\
\text { Investigación }\end{array}$ & $\begin{array}{c}\text { Población y } \\
\text { Muestra }\end{array}$ & Instrumentos & Aspectos & Resultados & Conclusión \\
\hline ético
\end{tabular}

\begin{tabular}{|c|c|c|c|c|c|c|}
\hline \multicolumn{7}{|c|}{ DATOS DE LA PUBLICACION } \\
\hline & 3. Autor & Año & \multicolumn{2}{|c|}{$\begin{array}{l}\text { Nombre de la } \\
\text { investigación }\end{array}$} & Revista donde se ubica la publicación & Volumen y número \\
\hline $\begin{array}{l}\text { Cabrera, } \\
\text { Díaz, Lidia; Bat }\end{array}$ & $\begin{array}{l}\text { ros, Otilia; } \\
\text { ros }\end{array}$ & 2019 & $\begin{array}{r}\text { La gestión de } \\
\text { territoriale } \\
\text { desarrollo so } \\
\text { las mon } \\
\text { de } \mathrm{C}\end{array}$ & $\begin{array}{l}\text { dicadores } \\
\text { para el } \\
\text { enible en } \\
\text { ñas } \\
\text { a }\end{array}$ & Fuente Académica Premier & Vol. 162 Issue 2, p1-18. $18 \mathrm{p}$ \\
\hline \multicolumn{7}{|c|}{ CONTENIDO DE LA PUBLICACIÓN } \\
\hline $\begin{array}{c}\text { Tipo de } \\
\text { Investigación }\end{array}$ & \multicolumn{2}{|c|}{$\begin{array}{l}\text { Población y } \\
\text { Muestra }\end{array}$} & Instrumentos & \multicolumn{2}{|c|}{$\begin{array}{cc}\text { Aspectos } & \text { Resultados } \\
\text { ético } & \\
\end{array}$} & Conclusión \\
\hline $\begin{array}{l}\text { prospectiva y } \\
\text { estadística }\end{array}$ & $\begin{array}{l}\text { Montañ } \\
\text { Guamuh }\end{array}$ & & $\begin{array}{c}\text { Análisis } \\
\text { Estructural }\end{array}$ & $\begin{array}{l}\text { Código } \\
\text { de ética } \\
\text { en } \\
\text { investig } \\
\text { ación }\end{array}$ & $\begin{array}{l}\text { Los resultados alcanzados indican que las } \\
37 \text { variables agrupadas en los factores } \\
\text { cuyas cargas factoriales son } \\
\text { considerablemente altas }(>0,75) \text {, pueden } \\
\text { ser consideradas como indicadores más } \\
\text { representativos del ecosistema y por } \\
\text { consiguiente los factores subyacentes } \\
\text { serán a efectos de este procedimiento los } \\
\text { factores clave. Ambos constituyen una } \\
\text { formación estructural consecuente para el } \\
\text { estudio de las dimensiones del desarrollo } \\
\text { sostenible en el ecosistema Guamuhaya. }\end{array}$ & $\begin{array}{l}\text { Los resultados obtenidos en } \\
\text { Guamuhaya } \\
\text { justifican la apremfuegos) } \\
\text { necesidad de realizar } \\
\text { transformaciones con mayor } \\
\text { grado de profundidad, para } \\
\text { lograr que esta región } \\
\text { montañosa exhiba todas sus } \\
\text { potencialidades y los } \\
\text { pobladores tengan mejor } \\
\text { calidad de vida con las } \\
\text { perspectivas de un futuro } \\
\text { próspero, para lo cual se } \\
\text { precisa la intervención }\end{array}$ \\
\hline
\end{tabular}


oportuna de las instituciones responsables con especial incidencia en las variables clave, de esta podrán revertirse los problemas $\mathrm{y}$ limitaciones lo que permitirá modificar la situación actual del ecosistema para aproximarse al estado más deseado.

\begin{tabular}{ccc}
\hline 4. Autor & Año & $\begin{array}{c}\text { Nombre de la } \\
\text { investigación }\end{array}$ \\
\hline $\begin{array}{c}\text { María A. Rodríguez } \\
\text { T. }\end{array}$ & 2014 & $\begin{array}{c}\text { Indicadores de gestión en la } \\
\text { gerencia estratégica } \\
\text { universitaria }\end{array}$ \\
\end{tabular}

\section{S DE LA PUBLICACIÓN}

Revista donde se ubica la publicación

Revista Científica Electrónica de Ciencias

Humanas / Scientific e-journal of Human

Sciences / PPX200502ZU1935 / ISSN

1856-1594 / By Fundación Unamuno /

Venezuela

www.revistaorbis.org.ve

\section{CONTENIDO DE LA PUBLICACIÓN}

\begin{tabular}{|c|c|c|c|c|c|}
\hline $\begin{array}{c}\text { Tipo de } \\
\text { Investigación }\end{array}$ & $\begin{array}{c}\text { Población y } \\
\text { Muestra }\end{array}$ & Instrumentos & $\begin{array}{c}\text { Aspectos } \\
\text { ético }\end{array}$ & Resultados & Conclusión \\
\hline Descriptivo & $\begin{array}{l}\text { La muestra } \\
\text { tomada fue de } \\
\text { los docentes, } \\
\text { estudiantes y } \\
\text { personal } \\
\text { administrativo, } \\
\text { de Universidad } \\
\text { de Carabobo un } \\
\text { total de } 160\end{array}$ & $\begin{array}{l}\text { Recolección de } \\
\text { información y } \\
\text { observación, a } \\
\text { través de } \\
\text { revisión } \\
\text { documental y } \\
\text { fichas de } \\
\text { observación y } \\
\text { cálculo. }\end{array}$ & $\begin{array}{l}\text { Código de } \\
\text { ética en } \\
\text { investigación }\end{array}$ & $\begin{array}{l}\text { A partir de un estudio teórico se } \\
\text { propone la definición y construcción } \\
\text { de indicadores de gestión, que } \\
\text { permitan el establecimiento de } \\
\text { mecanismos que garanticen el } \\
\text { seguimiento de ellos, permitiendo al } \\
\text { nivel directivo en cualquiera de los } \\
\text { niveles de la organización, la toma } \\
\text { de decisiones de manera acertada y } \\
\text { oportuna. }\end{array}$ & $\begin{array}{l}\text { El presente artículo realiza una } \\
\text { exposición sobre el compromiso que } \\
\text { tienen las universidades con la } \\
\text { sociedad, donde están llamadas actuar y } \\
\text { desarrollarse con pertinencia, } \\
\text { entendiéndose por pertinencia, una } \\
\text { visión holística donde no solo se } \\
\text { vincula con el aspecto académico y } \\
\text { profesional; también con la calidad, } \\
\text { equidad, responsabilidad social, } \\
\text { diversidad y el dialogo intercultural, }\end{array}$ \\
\hline
\end{tabular}


todo ello frente a las necesidades de las comunidades regionales, nacionales e internacionales donde conviven.

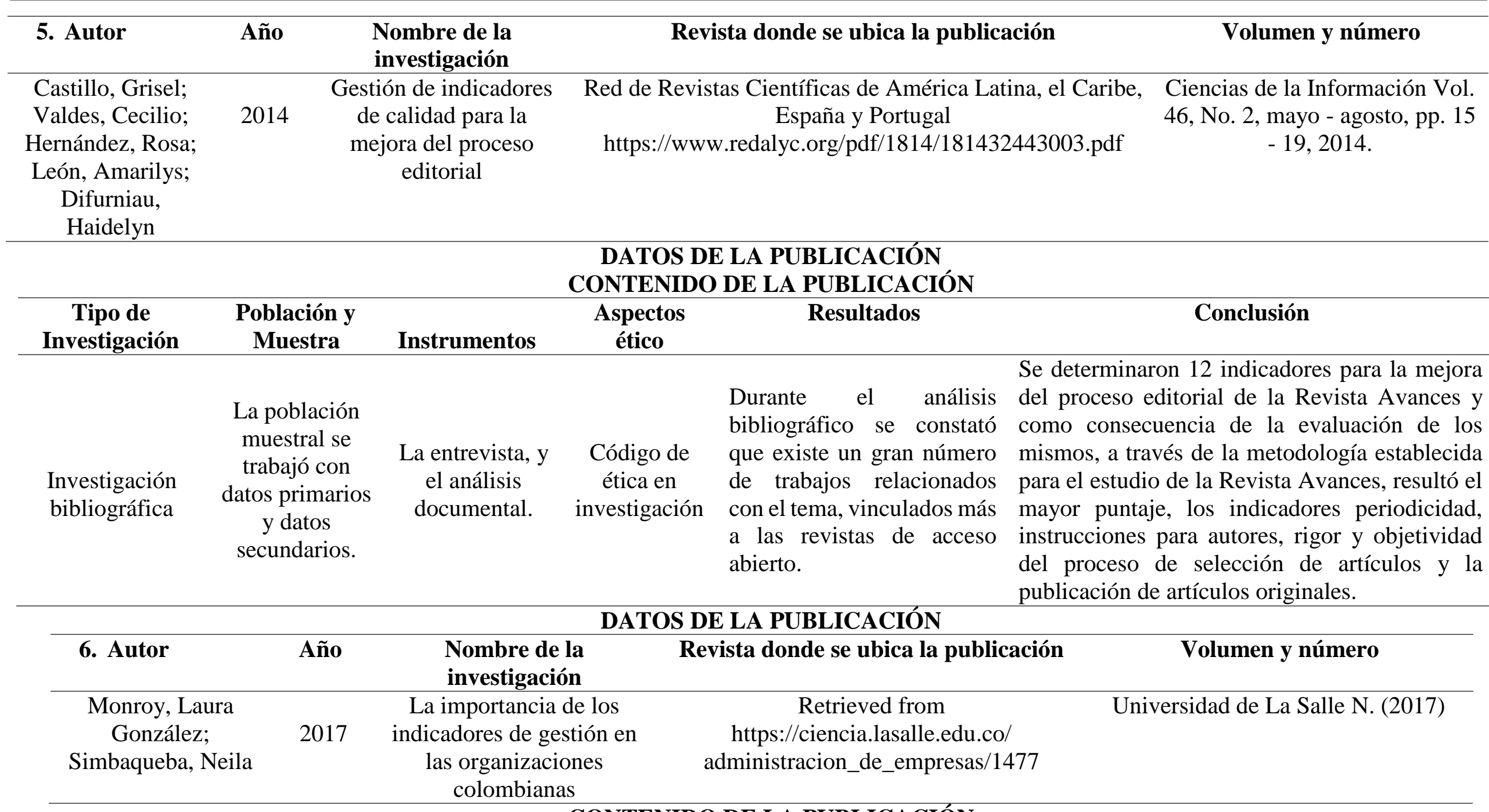

\section{CONTENIDO DE LA PUBLICACIÓN}




\begin{tabular}{|c|c|c|c|c|c|c|}
\hline $\begin{array}{c}\text { Tipo de } \\
\text { Investigación }\end{array}$ & \multicolumn{2}{|c|}{$\begin{array}{c}\text { Población y } \\
\text { Muestra }\end{array}$} & Instrumentos & \multicolumn{2}{|r|}{ Resultados } & Conclusión \\
\hline $\begin{array}{c}\text { Revisión } \\
\text { bibliográfica }\end{array}$ & $\begin{array}{r}\mathrm{Lap} \\
\mathrm{mu} \\
\text { trabaj } \\
\text { prima } \\
\text { secl }\end{array}$ & $\begin{array}{l}\text { blación } \\
\text { stral se } \\
\text { con datos } \\
\text { os y datos } \\
\text { adarios. }\end{array}$ & $\begin{array}{l}\text { La entrevista, y } \\
\text { el análisis } \\
\text { documental. }\end{array}$ & $\begin{array}{l}\text { Código de } \\
\text { ética en } \\
\text { investigación }\end{array}$ & $\begin{array}{l}\text { Se presenta una metodología sencilla y } \\
\text { clara para la construcción de } \\
\text { indicadores que agreguen valor a la } \\
\text { toma de decisiones en las } \\
\text { organizaciones. Para ello es necesario } \\
\text { mencionar aspectos tales como } \\
\text { definición, ventajas y desventajas, } \\
\text { tipos y elementos que los componen. }\end{array}$ & $\begin{array}{l}\text { Los indicadores de gestión son } \\
\text { una herramienta de un alto grado } \\
\text { de funcionalidad, puesto que } \\
\text { permite ser implementada en } \\
\text { una organización sin que sea } \\
\text { necesario tener en cuenta } \\
\text { aspectos como el tamaño, sector } \\
\text { o tipo de empresa. }\end{array}$ \\
\hline \multicolumn{7}{|c|}{ DATOS DE LA PUBLICACIÓN } \\
\hline 7. Autor & Año & \multicolumn{4}{|c|}{$\begin{array}{l}\text { Nombre de la Revista donde se ubica la publicación } \\
\text { investigación }\end{array}$} & Volumen y número \\
\hline $\begin{array}{l}\text { Romero , Ariel; } \\
\text { Alfonso, } \\
\text { Iruma; Latorre, } \\
\text { Luis }\end{array}$ & 2020 & \multicolumn{4}{|c|}{ 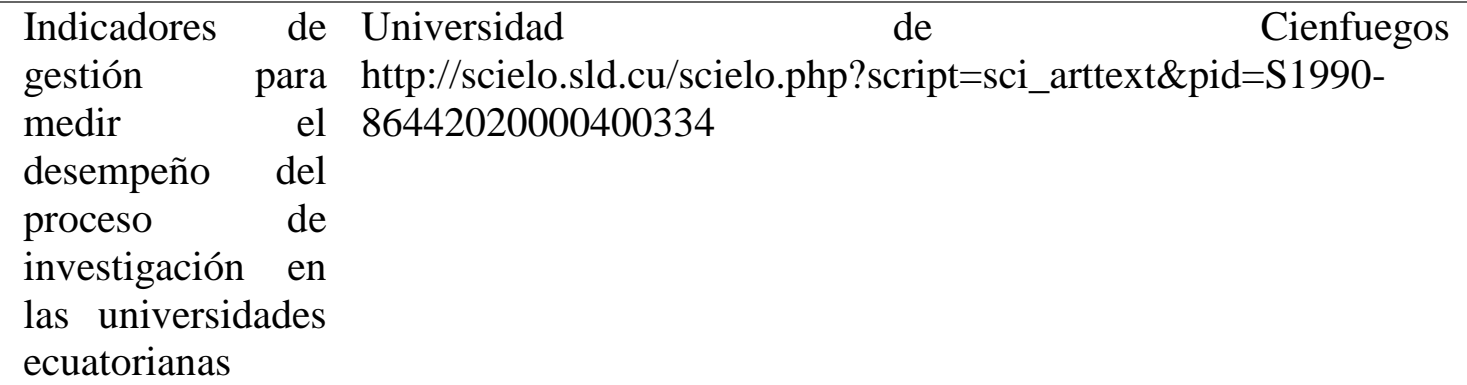 } & $\begin{array}{l}\text { vol.16 no.75 Cienfuegos jul.- } \\
\text { ago. } 2020 \text { Epub 02-Ago-2020 }\end{array}$ \\
\hline
\end{tabular}

\begin{tabular}{|c|c|c|c|c|c|}
\hline \multicolumn{6}{|c|}{$\begin{array}{c}\text { CONTENIDO DE LA PUBLICACION } \\
\end{array}$} \\
\hline $\begin{array}{c}\text { Tipo de } \\
\text { Investigación }\end{array}$ & $\begin{array}{c}\text { Población y } \\
\text { Muestra }\end{array}$ & Instrumentos & $\begin{array}{c}\text { Aspectos } \\
\text { ético }\end{array}$ & Resultados & Conclusión \\
\hline Descriptiva & $\begin{array}{l}\text { La muestra estuvo } \\
\text { conformada por } 16 \\
\text { coordinadores de la } \\
\text { función de } \\
\text { investigación. }\end{array}$ & $\begin{array}{c}\text { La entrevista, y } \\
\text { el análisis } \\
\text { documental. }\end{array}$ & $\begin{array}{l}\text { Código de } \\
\text { ética en } \\
\text { investigación }\end{array}$ & $\begin{array}{l}\text { El diagnóstico realizado sobre los } \\
\text { mecanismos empleados en las } \\
\text { universidades para evaluar el } \\
\text { desempeño del proceso de } \\
\text { investigación evidenció que, solo en } \\
\text { el } 56,2 \% \text { de las instituciones } \\
\text { estudiadas están definidos y } \\
\text { caracterizados los procesos que en } \\
\text { ellas se desarrollan, aspecto que }\end{array}$ & $\begin{array}{l}\text { El diagnóstico desarrollado } \\
\text { con } 16 \text { coordinadores de } \\
\text { investigación } \\
\text { universidades de las tres } \\
\text { regiones (Sierra, Costa y } \\
\text { Amazonía) evidenció que no } \\
\text { todas las instituciones tienen } \\
\text { identificados sus procesos, } \\
\text { que no siempre saben }\end{array}$ \\
\hline
\end{tabular}


coincide con los resultados clasificar los procesos obtenidos. universitarios, que no siempre se evalúa el impacto de la investigación $y$, que no siempre los mecanismos empleados permiten tener una valoración objetiva del desempeño investigación.

\begin{tabular}{|c|c|c|c|c|}
\hline \multicolumn{5}{|c|}{ DATOS DE LA PUBLICACIÓN } \\
\hline 8. Autor & Año & $\begin{array}{l}\text { Nombre de la } \\
\text { investigación }\end{array}$ & Revista donde se ubica la publicación & Volumen y número \\
\hline $\begin{array}{l}\text { Alfaro, César; } \\
\text { Gómez, Javier }\end{array}$ & 2016 & $\begin{array}{c}\text { Un sistema de } \\
\text { indicadores para la } \\
\text { medición, evaluación, } \\
\text { innovación y } \\
\text { participación orientado a } \\
\text { la administración pública }\end{array}$ & $\begin{array}{l}\text { methaodos.revista de ciencias sociales } \\
\text { https://www.redalyc.org/pdf/4415/441548188006.pdf }\end{array}$ & $\begin{array}{c}\text { vol. 4, núm. 2, 2016, pp. 274- } \\
290 \text { Universidad Rey Juan } \\
\text { Carlos Madrid, España }\end{array}$ \\
\hline
\end{tabular}

\begin{tabular}{cccc}
\hline $\begin{array}{c}\text { Tipo de } \\
\text { Investigación }\end{array}$ & $\begin{array}{c}\text { Población y } \\
\text { Muestra }\end{array}$ & Instrumentos & $\begin{array}{c}\text { Aspectos } \\
\text { ético }\end{array}$ \\
\hline Descriptiva & $\begin{array}{c}\text { La población } \\
\text { muestral se trabajó } \\
\text { con datos primarios } \\
\text { y datos secundarios }\end{array}$ & $\begin{array}{c}\text { La entrevista, y } \\
\text { el análisis } \\
\text { documental. }\end{array}$ & $\begin{array}{c}\text { Código de } \\
\text { ética en } \\
\text { investigación }\end{array}$ \\
& & & \\
& &
\end{tabular}

El presente artículo perfila un Con esta aproximación a un sistema tecnológico de medición y sistema de indicadores para la evaluación basado en indicadores y medición, evaluación, orientado a la Administración innovación y participación Pública. El sistema estará enfocado orientado a la Administración a un modelo colaborativo que Pública, pretendemos aportar fomente la participación ciudadana una herramienta que, junto a y promueva la innovación social otras muchas en el marco de para el desarrollo de productos o una nueva cultura de la procesos novedosos que, estando gobernanza, contribuya a dar orientados a la resolución de los respuesta a la intensidad de los problemas más acuciantes de los cambios que generan las 
ciudadanos, supongan una mejora y renovadas expectativas y transformación del entorno social y demandas sociales, así como a de las relaciones humanas. los nuevos problemas $\mathrm{y}$ desafíos en la gestión de los asuntos públicos.

\section{DATOS DE LA PUBLICACIÓN}

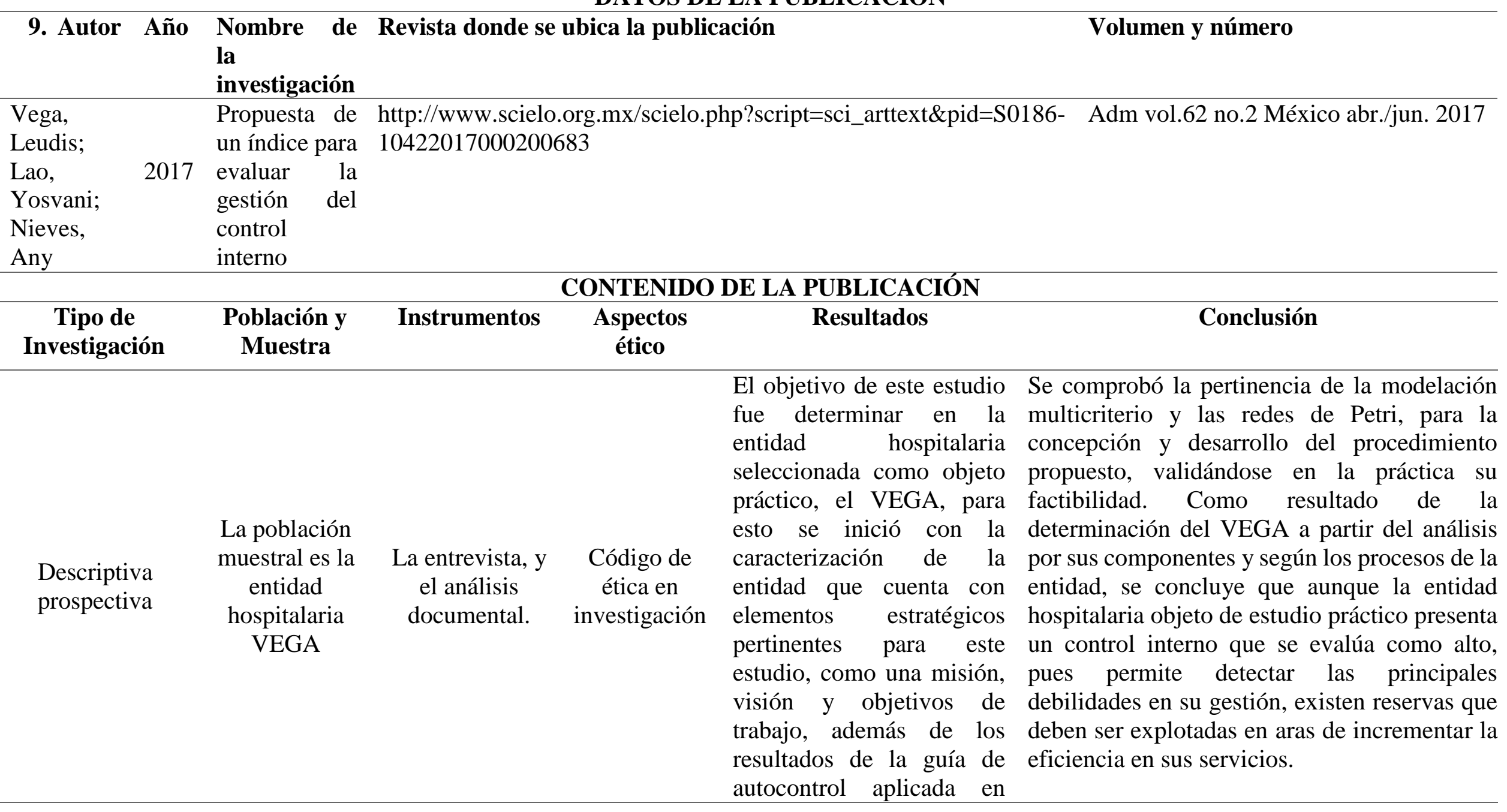


julio del 2015, como

resultado de una auditoría

externa.

\begin{tabular}{|c|c|c|c|c|c|}
\hline \multicolumn{6}{|c|}{ DATOS DE LA PUBLICACIÓN } \\
\hline 10. Autor & Año & $\begin{array}{l}\text { Nombre de la } \\
\text { investigación }\end{array}$ & \multicolumn{2}{|c|}{ Revista donde se ubica la publicación } & Volumen y número \\
\hline $\begin{array}{l}\text { Comas, Raul; } \\
\text { Montes, Jimena; } \\
\text { Moreno, Milton; } \\
\text { Valle, Betty }\end{array}$ & $\begin{array}{r}\text { her } \\
\text { contr } \\
\mathrm{d} \\
\mathrm{c}\end{array}$ & $\begin{array}{l}\text { ntegración de } \\
\text { amientas para el } \\
\text { ol de gestión. Caso } \\
\text { estudio en una } \\
\text { empresa } \\
\text { mercializadora }\end{array}$ & \multicolumn{2}{|c|}{$\begin{array}{c}\text { Revista de Ciencia, Tecnología e Innovación. ISSN 1390- } \\
9150 \\
\text { Dialnet- } \\
\text { IntegracionDeHerramientasParaElControlDeGestionCas- } \\
6756402 \% 20 \text {.pdf }\end{array}$} & $\begin{array}{l}\text { Vol. 5, Núm. 2, pp. 101- } \\
\text { 114, abril-junio } 2018 .\end{array}$ \\
\hline \multicolumn{6}{|c|}{ CONTENIDO DE LA PUBLICACIÓN } \\
\hline $\begin{array}{c}\text { Tipo de } \\
\text { Investigación }\end{array}$ & $\begin{array}{l}\text { Población y } \\
\text { Muestra }\end{array}$ & Instrumentos & $\begin{array}{l}\text { Aspectos } \\
\text { ético }\end{array}$ & Resultados & Conclusión \\
\hline $\begin{array}{l}\text { Descriptiva - } \\
\text { Prospectiva }\end{array}$ & $\begin{array}{c}\text { La población } \\
\text { muestral se } \\
\text { trabajó con el } \\
\text { personal de la } \\
\text { empresa } \\
\text { comercializador } \\
\text { a }\end{array}$ & $\begin{array}{l}\text { La entrevista, y } \\
\text { el análisis } \\
\text { documental. }\end{array}$ & $\begin{array}{l}\text { Código de } \\
\text { ética en } \\
\text { investigación }\end{array}$ & 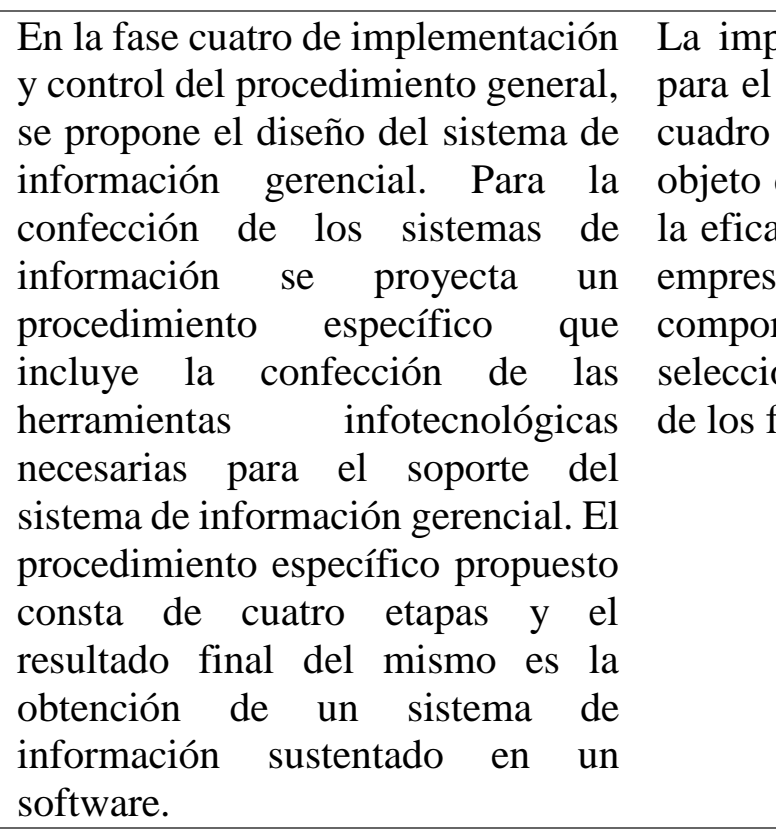 & $\begin{array}{l}\text { nentación del procedimiento } \\
\text { ntrol de gestión basado en el } \\
\text { mando integral en la empresa } \\
\text { estudio contribuyó a mejorar } \\
\text { y la eficiencia de la gestión } \\
\text { al, al mejorar el } \\
\text { miento de los indicadores } \\
\text { dos para medir el desempeño } \\
\text { ores claves de éxito. }\end{array}$ \\
\hline
\end{tabular}




\section{DISCUSIÓN}

Posterior a la revisión sistemática de los artículos, encontramos que del 100\% corresponde a América latina y el Caribe, resaltando el país de Chile con el 30\% de publicaciones respectivamente, seguido de los países de Cuba, Colombia, Ecuador, Argentina, Venezuela y Brasil que alcanzaron 10\% cada uno. En cuanto a los diseños y tipos de estudios el 100\% son descriptivos con sus diferentes variantes.

En concordancia a los indicadores de gestión podemos destacar que Huerta, Patricia, establece que, Los indicadores de riesgos para la salud se caracterizaron principalmente porque en un 50\% y más de las regiones analizadas obtuvieron un resultado similar al resultado esperado. A diferencia de los indicadores de estadísticas vitales, en donde un $49 \%$ y menos de las regiones tuvieron un resultado similar al esperado en el mismo periodo. También Cerdá Suárez, L.; Santibáñez Vivanco, A; Asensio del Arco, E; Martínez A., Enfatizan la importancia de intervenir sobre ciertas variables relevantes en diferentes niveles de actuación de una unidad hospitalaria. Por su parte Cabrera, Elia; Díaz, Lidia; Barros, Otilia, indica que los resultados obtenidos en Guamuhaya (Cienfuegos) justifican la apremiante necesidad de realizar transformaciones con mayor grado de profundidad, para lograr que esta región montañosa exhiba todas sus potencialidades y los pobladores tengan mejor calidad de vida con las perspectivas de un futuro próspero, para lo cual se precisa la intervención oportuna de las instituciones responsables con especial incidencia en las variables clave, de esta podrán revertirse los problemas y limitaciones lo que permitirá modificar la situación actual del ecosistema para aproximarse al estado más deseado.

Por otro lado, María A. Rodríguez T. su artículo realiza una exposición sobre el compromiso que tienen las universidades con la sociedad, donde están llamadas actuar y desarrollarse con pertinencia, entendiéndose por pertinencia, una visión holística donde no solo se vincula con el aspecto académico y profesional; también con la calidad, equidad, responsabilidad social, diversidad y el dialogo intercultural, todo ello frente a las necesidades de las comunidades regionales, nacionales e internacionales donde conviven. Por su parte Castillo, Grisel; Valdes, Cecilio; Hernández, Rosa; León, Amarilys; Difurniau, Haidelyn, establecen que, Se determinaron 12 indicadores para la mejora del proceso editorial de la Revista Avances y como consecuencia de la evaluación de los mismos, a través de la metodología establecida para el estudio de la Revista Avances, resultó el mayor puntaje, los indicadores periodicidad, instrucciones para autores, rigor 
y objetividad del proceso de selección de artículos y la publicación de artículos originales. También Monroy, Laura González; Simbaqueba, Neila, establecieron una metodología sencilla y clara para la construcción de indicadores que agreguen valor a la toma de decisiones en las organizaciones. Para ello es necesario mencionar aspectos tales como definición, ventajas y desventajas, tipos y elementos que los componen.

También, Romero, Ariel; Alfonso, Iruma; Latorre, Luis, establecen que, el diagnóstico realizado sobre los mecanismos empleados en las universidades para evaluar el desempeño del proceso de investigación evidenció que, solo en el 56,2\% de las instituciones estudiadas están definidos y caracterizados los procesos que en ellas se desarrollan, aspecto que coincide con los resultados obtenidos. Asimismo, Alfaro, César; Gómez, Javier, determinó una, aproximación a un sistema de indicadores para la medición, evaluación, innovación y participación orientado a la Administración Pública, pretendemos aportar una herramienta que, junto a otras muchas en el marco de una nueva cultura de la gobernanza, contribuya a dar respuesta a la intensidad de los cambios que generan las renovadas expectativas y demandas sociales, así como a los nuevos problemas y desafíos en la gestión de los asuntos públicos.. Igualmente, Vega, Leudis; Lao, Yosvani; Nieves, Any, indican que, se comprobó la pertinencia de la modelación multicriterio y las redes de Petri, para la concepción y desarrollo del procedimiento propuesto, validándose en la práctica su factibilidad. Como resultado de la determinación del VEGA a partir del análisis por sus componentes y según los procesos de la entidad, se concluye que aunque la entidad hospitalaria objeto de estudio práctico presenta un control interno que se evalúa como alto, pues permite detectar las principales debilidades en su gestión, existen reservas que deben ser explotadas en aras de incrementar la eficiencia en sus servicios.

Además, Comas,Raul; Montes, Jimena; Moreno, Milton; Valle, Betty, determinaron que, la implementación del procedimiento para el control de gestión basado en el cuadro de mando integral en la empresa objeto de estudio contribuyó a mejorar la eficacia y la eficiencia de la gestión empresarial, al mejorar el comportamiento de los indicadores seleccionados para medir el desempeño de los factores claves de éxito.

\section{CONCLUSIÓN O CONSIDERACIONES FINALES}

La Gestión y control de indicadores debe reportarse en forma oportuna. Un indicador debe contar con datos actualizados según la fecha y la hora, debe ser revisado y analizado. Con esto se 
establece que el indicador permite tomar decisiones para anticiparse o reaccionar de forma rápida y efectiva según los problemas y las oportunidades.

Un sistema de medición de indicadores de desempeño es una herramienta indispensable para ese fin. En el ámbito de la gestión de procesos se usa mucho la frase: "No se puede mejorar lo que no se puede medir". De esta manera el sistema de indicadores contribuye a alinear a toda la organización hacia una misma meta.

El propósito principal de trabajar con una metodología en la definición de indicadores es asegurar que los resultados sean significativos o relevantes para la empresa, es decir, que los indicadores que se utilizan sirvan de forma efectiva para alinear el desempeño hacia los objetivos de la empresa.

\section{LISTA DE REFERENCIAS}

Patricia Carolina Huerta Riveros Dirección: Avenida Collao 1202, Casilla 5-C, Universidad del Bío-Bío, Concepción-

Chile.http://revistas.ujat.mx/index.php/horizonte/article/view/2751

Cerdá Suárez LM, Santibáñez Vivanco A, Asensio del Arco E, Martínez Martínez A. http://eds.b.ebscohost.com/eds/detail/detail?vid=4\&sid=1572f6b9-1012-458f92f6-7de55d14c181\%40pdcvsessmgr06\&bdata=Jmxhbmc9ZXMmc210ZT11ZHMtbG12ZQ\%3d\%3d\#AN=135057266\&db =fua

Elia Natividad Cabrera Álvarez - Lidia Inés Díaz Gispert - Otilia Barros Díaz http://eds.b.ebscohost.com/eds/detail/detail?vid=7\&sid=1572f6b9-1012-458f-92f6$7 \mathrm{de} 55 \mathrm{~d} 14 \mathrm{c} 181 \% 40 \mathrm{pdc}-\mathrm{v}-$ sessmgr06\&bdata=Jmxhbmc9ZXMmc210ZT11ZHMtbGl2ZQ\%3d\%3d\#AN=139381 $287 \& \mathrm{db}=$ fua

Rodríguez T, María A. Indicadores de gestión en la gerencia estratégica universitaria Orbis. Revista Científica Ciencias Humanas, vol. 9, núm. 27, enero-abril, 2014, pp. 31-46 Fundación Miguel Unamuno y Jugo Maracaibo, Venezuela

Castillo Almeida, Grisel; Valdes García, Cecilio; Hernández Martínez, Rosa A.; León Paredes, Amarilys de la C.; Difurniau Gráu, Haidelyn Gestión de indicadores de calidad para la mejora del proceso editorial Ciencias de la Información, vol. 45, núm. 2, mayo- 
agosto, 2014, pp. 15-19 Instituto de Información Científica y Tecnológica La Habana, Cuba. https://www.redalyc.org/pdf/1814/181432443003.pdf

Monroy González, L. L., \& Simbaqueba Prieto, N. (2017). La importancia de los indicadores de gestión en las organizaciones colombianas. Retrieved from https://ciencia.lasalle.edu.co/ administracion_de_empresas/1477

Romero, Ariel; Alfon so, Iruma; Latorre, Luis. Indicadores de gestión para medir el desempeño del proceso de investigación en las universidades ecuatorianasConrado vol.16 no.75 Cienfuegos jul.-ago. 2020 Epub 02-Ago-2020. http://scielo.sld.cu/scielo.php?script=sci_arttext\&pid=S1990-86442020000400334

Alfaro, César; Gómez, Javier Un sistema de indicadores para la medición, evaluación, innovación y participación orientado a la administración pública methaodos.revista de ciencias sociales, vol. 4, núm. 2, 2016, pp. 274-290 Universidad Rey Juan Carlos Madrid, España. https://www.redalyc.org/pdf/4415/441548188006.pdf

Vega, Leudis; Lao, Yosvani; Nieves, Any. Propuesta de un índice para evaluar la gestión del control interno. Adm vol.62 no.2 México abr./jun. 2017. http://www.scielo.org.mx/scielo.php?script=sci_arttext\&pid=S018610422017000200683

Comas,Raul; Montes, Jimena; Moreno, Milton; Valle, Betty. Integración de herramientas para el control de gestión. Caso de estudio en una empresa comercializadora. Revista de Ciencia, Tecnología e Innovación. ISSN 1390-9150 Comas Rodríguez. R., Montes de Oca Sánchez, J. E., Moreno Villacis, M.D., Valle Fiallos, B. G. Vol. 5, Núm. 2, pp. 101-114, abril-junio 2018. 